ARTICLE

Received 22 Aug 2015 | Accepted 19 Feb 2016 | Published 31 Mar 2016

DOI: $10.1038 /$ ncomms11095

OPEN

\title{
Identification of plant vacuolar transporters mediating phosphate storage
}

Tzu-Yin Liu ${ }^{1, \star}, \uparrow$, Teng-Kuei Huang ${ }^{1,2,3, \star}$, Shu-Yi Yang ${ }^{1, \star}$, Yu-Ting Hong ${ }^{1}$, Sheng-Min Huang ${ }^{4}$, Fu-Nien Wang ${ }^{4}$, Su-Fen Chiang ${ }^{1}$, Shang-Yueh Tsai ${ }^{5}$, Wen-Chien Lu' ${ }^{1} \&$ Tzyy-Jen Chiou, ${ }^{1,2,6}$

Plant vacuoles serve as the primary intracellular compartments for inorganic phosphate ( $\mathrm{Pi}$ ) storage. Passage of $\mathrm{Pi}$ across vacuolar membranes plays a critical role in buffering the cytoplasmic Pi level against fluctuations of external $\mathrm{Pi}$ and metabolic activities. Here we demonstrate that the SPX-MFS proteins, designated as PHOSPHATE TRANSPORTER 5 family (PHT5), also named Vacuolar Phosphate Transporter (VPT), function as vacuolar Pi transporters. Based on ${ }^{31} \mathrm{P}$-magnetic resonance spectroscopy analysis, Arabidopsis pht5;1 loss-of-function mutants accumulate less $\mathrm{Pi}$ and exhibit a lower vacuolar-to-cytoplasmic $\mathrm{Pi}$ ratio than controls. Conversely, overexpression of PHT5 leads to massive Pi sequestration into vacuoles and altered regulation of Pi starvation-responsive genes. Furthermore, we show that heterologous expression of the rice homologue OsSPX-MFS1 mediates Pi influx to yeast vacuoles. Our findings show that a group of $\mathrm{Pi}$ transporters in vacuolar membranes regulate cytoplasmic Pi homeostasis and are required for fitness and plant growth.

\footnotetext{
${ }^{1}$ Agricultural Biotechnology Research Center, Academia Sinica, No. 128, Academia Road, Section 2, Taipei 11529, Taiwan. ${ }^{2}$ Molecular and Biological Agricultural Sciences Program, Taiwan International Graduate Program, Academia Sinica, Taipei 11529, Taiwan. ${ }^{3}$ Graduate Institute of Biotechnology, National Chung-Hsing University, Taichung 40243, Taiwan. ${ }^{4}$ Department of Biomedical Engineering and Environmental Sciences, National Tsing Hua University, Hsinchu 30013, Taiwan. ${ }^{5}$ Graduate Institute of Applied Physics, National Chengchi University, Taipei 11605, Taiwan. ${ }^{6}$ Biotechnology Center, National ChungHsing University, Taichung 40243, Taiwan. ${ }^{\star}$ These authors contributed equally to this work. † Present address: Department of Life Science and Institute of Bioinformatics and Structural Biology, National Tsing Hua University, Hsinchu 30013, Taiwan. Correspondence and requests for materials should be addressed to T.-J.C. (email: tjchiou@gate.sinica.edu.tw).
} 
P hosphorus (P) acquired in the form of inorganic phosphate (Pi) is one of the most abundant macronutrients in plant tissues ${ }^{1,2}$. Owing to its chemical properties, $\mathrm{Pi}$ forms insoluble complexes or precipitates with organic matter or mineral cations that are easily immobilized in the soil, rendering $\mathrm{Pi}$ availability a limiting factor for plant growth and development ${ }^{3}$. To ensure crop productivity, farmers apply large quantities of $\mathrm{Pi}$ fertilizers produced from non-renewable rock phosphate. Concern over the gradual depletion of global $\mathrm{P}$ reserves and the increasing demand for high crop yields due to increasing world population over the past few decades has resulted in a need to better understand how to develop crop varieties that use $\mathrm{Pi}$ more efficiently, thereby cutting down the huge costs incurred by fertilizer consumption and providing a means to achieve sustainable agriculture ${ }^{4}$.

Grown under varying conditions of $\mathrm{Pi}$ availability, plants coordinate discrete $\mathrm{Pi}$ transport activities across membranes, to maintain the cellular $\mathrm{Pi}$ homeostasis required for metabolic regulation and signal transduction ${ }^{5}$. For example, members of the PHOSPHATE TRANSPORTER 1 (PHT1) family, localized in the plasma membranes, are responsible for the external Pi acquisition and/or Pi translocation between cells or tissues ${ }^{6}$, whereas the members of the PHT2, PHT3 and PHT4 families belong to the organelle Pi transporters, targeted to mitochondria, plastids or Golgi for energy metabolism and stress responses ${ }^{7-12}$.

The vacuole, which occupies most of the volume of the plant cell, serves as a primary intracellular compartment for storage and remobilization of $\mathrm{Pi}^{13}$. Under adequate $\mathrm{Pi}$ supply, $\sim 70-95 \%$ of the intracellular $\mathrm{Pi}$ is stored in the vacuole ${ }^{14}$. When external $\mathrm{Pi}$ is in scarce supply, the level of cytoplasmic Pi (cyt-Pi; the sum of $\mathrm{Pi}$ in the cytosol and the non-vacuole organelles) is kept relatively constant at the expense of vacuolar Pi (vac-Pi) (refs 15,16), whereas when $\mathrm{Pi}$ is resupplied to $\mathrm{Pi}$-starved plants, rapid and massive $\mathrm{Pi}$ uptake to the plasma membrane is accompanied by the efficient sequestration of $\mathrm{Pi}$ inside the vacuole ${ }^{17,18}$. Despite the critical role of vac-Pi in buffering the cyt-Pi against fluctuations caused by variable $\mathrm{Pi}$ availability and metabolic activities, the molecular identity of the vac-Pi transporter as well as the regulatory mechanism by which $\mathrm{Pi}$ is translocated across vacuolar membranes (tonoplast) remain elusive.

Several eukaryotic SYG1/PHO81/XPR1 (SPX) domaincontaining proteins have been implicated in the regulation of $\mathrm{Pi}$ signalling and transport ${ }^{19,20}$, including the yeast low-affinity Pi transporters Pho87, Pho90 and Pho91 (refs 21,22). In plants, SPX domain-containing proteins can be classified into different families based on additional domains at the carboxyl terminus ${ }^{23,24}$. One of them possesses the major facilitator superfamily (MFS) domain that is found in many transporters mediating the translocation of small solutes, including $\mathrm{Pi}^{25}$. We therefore envisaged that the plant SPX-MFS family potentially functions as a new group of $\mathrm{Pi}$ transporters in plants.

Although the three Arabidopsis SPX-MFS proteins (At1g63010, At4g11810 and At4g22990) share only low sequence similarity (below 25\%) with the other known Arabidopsis Pi transporters, the capability of SPX-MFS proteins to transport Pi was assumed based on the observations of partial complementation of the Pi uptake-defective yeast mutants by the rice OsSPX-MFS1 or OsSPX-MFS3 and heterologous expression of OsSPX-MFS3 at the plasma membranes of Xenopus oocytes ${ }^{26,27}$. However, their transport properties and physiological roles in plants are unclear. In this study, we identified the Arabidopsis SPX-MFS proteins as the transporters mediating vac-Pi storage. Using in vivo ${ }^{31} \mathrm{P}$-magnetic resonance spectroscopy (MRS) analysis, we demonstrate that AtSPX-MFS1 loss-of-function mutants exhibit a lower vac/cyt-Pi ratio than wild-type (WT) plants, whereas overexpression of AtSPX-MFS genes leads to misregulation of $\mathrm{Pi}$ starvation-responsive (PSR) genes and growth retardation as a consequence of massive $\mathrm{Pi}$ sequestration into vacuoles. We also show the capability of the rice homologue OsSPX-MFS1 to mediate $\mathrm{Pi}$ influx into yeast vacuoles. The Arabidopsis SPX-MFS proteins are thus designated here as members of the PHOSPHATE TRANSPORTER 5 family (PHT5) following the systematic nomenclature of PHT1-PHT4 Pi transporters in Arabidopsis ${ }^{28,29}$. PHT5;1 was also named Vacuolar Phosphate Transporter 1 (VPT1) in a very recent article ${ }^{30}$. Our findings shed light on the crucial role of SPX-MFS in plant adaptation to Pi fluctuations and provide a new direction to explore the mechanisms by which plants modulate cyt-Pi homeostasis in response to varying $\mathrm{Pi}$ availability through understanding of the regulation of vac-Pi transporters.

\section{Results}

Arabidopsis PHT5 proteins reside in the vacuolar membrane. The Arabidopsis PHT5 family consists of three members, namely AtPHT5;1 (At1g63010), AtPHT5;2 (At4g11810) and AtPHT5;3 (At4g22990). To characterize their function, we first determined the subcellular localization of these proteins by fusing them with green fluorescent protein (GFP) and expressing them in Arabidopsis mesophyll protoplasts (Fig. 1a and Supplementary Fig. 1a,b), tobacco (Nicotiana benthamiana) leaves (Fig. 1b and Supplementary Fig. 1c,d) and Arabidopsis plants (Fig. 1c) under the control of the cauliflower mosaic virus $35 \mathrm{~S}$ promoter. The fluorescent labelling of all three GFP fusion proteins coincided with the tonoplast. The observation of the tonoplast localization of these proteins with potential as transporters prompted us to postulate that they might be the long-sought-after vac-Pi transporters. Further analyses of promoter activities using the $\beta$-glucuronidase (GUS) reporter system indicated that the three PHT5 genes displayed distinct but partially overlapping expression patterns. PHT5; 1 was ubiquitously expressed in most tissues (Fig. 1d-f) and PHT5;2 expression was confined to guard cells, vascular tissue and pollen (Fig. 1g-i). PHT5;3 showed a similar expression pattern to $P H T 5 ; 1$, but in the root its expression was exclusive to the stele (Fig. $1 \mathrm{j}-1$ ). The results of quantitative reverse transcription-PCR (qRT-PCR) showed that the PHT5;1 transcript was the most abundant among the gene family and the expression level of PHT5;2 was the lowest and hardest to detect. In shoots, the level of PHT5;1 and PHT5;3 transcript was independent of Pi status (Fig. 1m,n). In roots, although PHT5;1 was upregulated in response to Pi deficiency, PHT5;3 was downregulated (Fig. $1 \mathrm{~m}, \mathrm{n}$ ). Consistent with these results, the differences in the expression level of PHT5 genes were also observed in the subsequent RNA sequencing (RNA-seq) analyses (Supplementary Data 1).

Loss of PHT5; 1 leads to impaired Pi accumulation. We then analysed the Arabidopsis T-DNA insertion lines for the three respective PHT5 genes (Supplementary Fig. 2a-d). Under Pisufficient conditions, the pht5;1-1 knockdown mutant, as well as the pht5;1-2 and pht5;1-3 knockout mutants, led to a decrease in Pi levels up to $40 \%$ compared with the WT plants (Fig. 2a). By contrast, neither the pht5;2 nor the pht5;3, nor the pht5;2 pht5;3 double mutants showed an altered Pi level (Fig. 2a,b), suggesting that PHT5;1 plays a prominent role in Pi accumulation. Likewise, the double and the triple mutants lacking PHT5;1 but not the pht5;2 pht5;3 double mutant showed a reduced Pi level (Fig. 2b). The phenotype of pht5;1 mutants could be restored by the expression of PHT5;1 driven by the native promoter (Supplementary Fig. 2e), indicating that the reduction of the Pi level resulted from the loss of PHT5;1. 

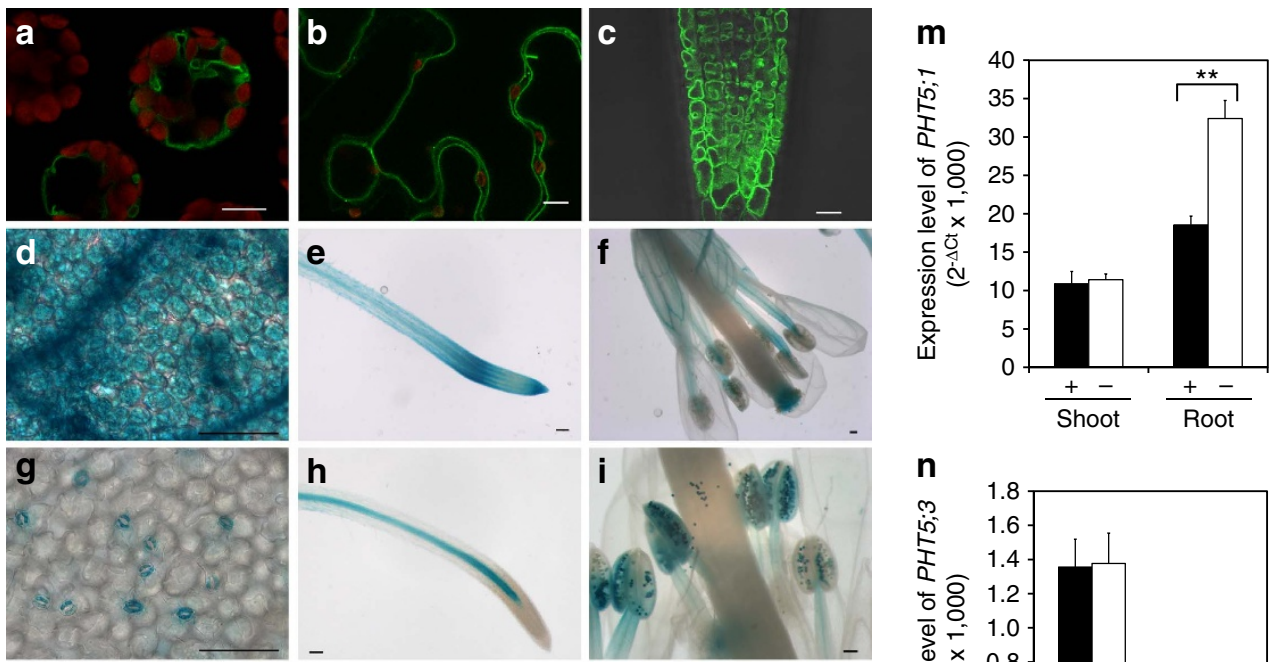

h
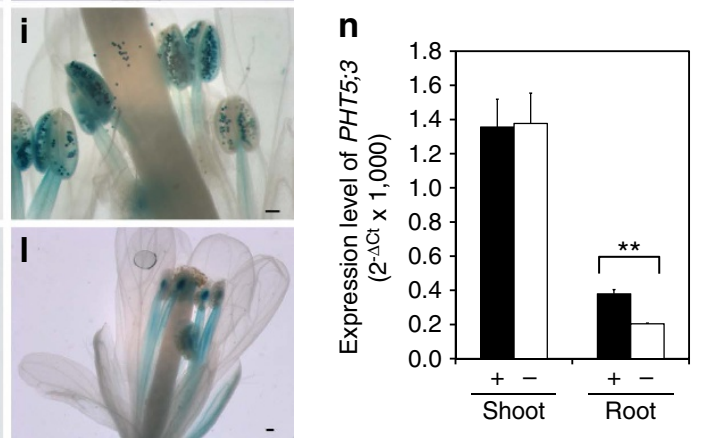

Figure 1 | Expression and tonoplast localization of AtPHT5 family members. Expression of p35S:PHT5;1-GFP in Arabidopsis mesophyll protoplasts (a), tobacco (N. benthamiana) leaves (b) and roots of Arabidopsis transgenic plants (c). Expression of pPHT5;1:GUS (d-f), pPHT5;2:GUS (g-i) and pPHT5;3:GUS $(\mathbf{j}-\mathbf{I})$ in 12-day-old seedlings grown under Pi-sufficient conditions $(\mathbf{d}, \mathbf{e}, \mathbf{g}, \mathbf{h}, \mathbf{j}, \mathbf{k})$ and in flowers of transgenic plants grown in soils $(\mathbf{f}, \mathbf{i}, \mathbf{l})$. Scale bars, $10 \mu \mathrm{m}$ $(\mathbf{a}, \mathbf{b}, \mathbf{c}) ; 100 \mu \mathrm{m}(\mathbf{d}-\mathbf{I})$. qRT-PCR analysis of PHT5;1 ( $\mathbf{m})$ and PHT5;3 (n) expression in the shoot and root of 13-day-old WT seedlings under Pi-sufficient ( + ) and Pi-deficient $\left(-, 7\right.$ days of Pi starvation) conditions. Error bar, s.e. $(n=3) .{ }^{\star \star} P<0.01$, Student's $t$-test. Results of qRT-PCR were reproducible in three independent experiments.

As the majority of the cellular Pi is stored in the vacuole, the reduction of the Pi level in the pht5;1 mutant can probably be attributed to the decreased accumulation of vac-Pi. We then investigated whether PHT5 family members mediate the sequestration of the bulk of cyt-Pi during $\mathrm{Pi}$ replenishment. The loss-of-function mutants of PHT5;1 (pht5;1-2, pht5;1-2 pht5;2, pht5;1-2 pht5;3 and pht5;1-2 pht5;2 pht5;3) developed severe leaf necrosis during Pi recovery following deficiency (Fig. 2c). As massive flux of $\mathrm{Pi}$ is transported into the cell on Pi replenishment, an efficient sequestration of Pi into the vacuole would be essential for the maintenance of cyt-Pi level in a physiological range. It is likely that loss of PHT5;1 impairs such activity, leading to the accumulation of cyt-Pi up to a toxic level and thus the necrosis at leaf margins as a symptom of Pi toxicity ${ }^{31}$. These observations suggest that PHT5;1 has an important role in adaptation to Pi fluctuations.

Overexpression of PHT5 leads to Pi overaccumulation. The results from the analysis of pht5;1 mutants suggested a potential role for PHT5 in mediating Pi import to the vacuole. To further examine this possibility, we analysed the transgenic plants overexpressing PHT5 proteins. In contrast to the pht5;1 mutant lines, the overexpression of PHT5;1-GFP resulted in $\mathrm{Pi}$ overaccumulation (Fig. 3a). The PHT5;2-HA- or PHT5;3-GFP-overexpressing lines also exhibited a higher level of Pi (Fig. 3b,c), indicating that the PHT5 members function similarly. In addition, the PHT5;1-GFP-overexpressing lines, as well as PHT5; 2-HA- and PHT5;3-GFP-overexpressors, showed retarded growth as reflected in an $\sim 50 \%$ reduction of the fresh weight compared with WT plants (Fig. 3d,e and Supplementary Fig. 3). We inferred that the overexpression of PHT5 proteins sequesters $\mathrm{Pi}$, thus stopping it from being used in the cytoplasm and leading to stunted growth.

PHT5 mediates Pi influx into vacuoles. To address whether PHT5 proteins directly contribute to the vacuolar storage of $\mathrm{Pi}$, we applied ${ }^{31} \mathrm{P}$-MRS-based analysis associated with magnetic resonance image. This technique, which was adapted from nuclear magnetic resonance (NMR) and is capable of differentiating between the vac-Pi and cyt-Pi pools by a chemical shift, owing to the different $\mathrm{pH}$ environments ${ }^{32}$, allows the noninvasive analysis of intact living plants without the circulating perfusion system. After $\sim 1 \mathrm{~h}$ of scan time, the two resolvable peaks corresponding to vac-Pi and cyt-Pi in the Arabidopsis WT whole seedlings were resolved at $1.1 \pm 0.09$ p.p.m. $( \pm$ s.d., $n=6)$ and $2.4 \pm 0.11$ p.p.m. $(n=6)$, respectively (Fig. $4 a)$. The vac-Pi and cyt-Pi levels of the pht5;1-2, the triple mutants, the PHT5 overexpressors and the $\mathrm{Pi}$ overaccumulator pho2 mutant $\mathrm{t}^{33,34}$ were inspected under the same conditions (Fig. 4a). The vac/cyt Pi ratio of WT seedlings was calculated to be $5.6 \pm 1.2(n=6)$, referring the distribution of $84 \%$ of $\mathrm{Pi}$ in the vacuole and $16 \%$ of $\mathrm{Pi}$ in the cytoplasm (Fig. 4b). Although the pht5;1-2 exhibited a lower ratio of vac/cyt $\mathrm{Pi}(2.7 \pm 0.6)( \pm$ s.d., $n=7)$ relative to WT plants, the PHT5 overexpressor had a higher ratio $(15.6 \pm 5.8$ for PHT5;2-HA, $\quad n=12 ; \quad 10.6 \pm 4.4 \quad$ for PHT5;1-GFP, $n=6$; Fig. $4 \mathrm{~b}$ ). Remarkably, the vac-Pi peak of the triple mutant was nearly abolished with an extremely low vac/cyt Pi $(0.8 \pm 0.2$, $n=4$ ). These results suggest that PHT5 proteins regulate the vacuolar compartmentation of $\mathrm{Pi}$ and are functionally redundant.

Using the same batch of seedlings subjected to MRS analysis, in parallel we measured the total Pi level of the plants and calculated the subcellular $\mathrm{Pi}$ distribution between the vacuoles and 
a

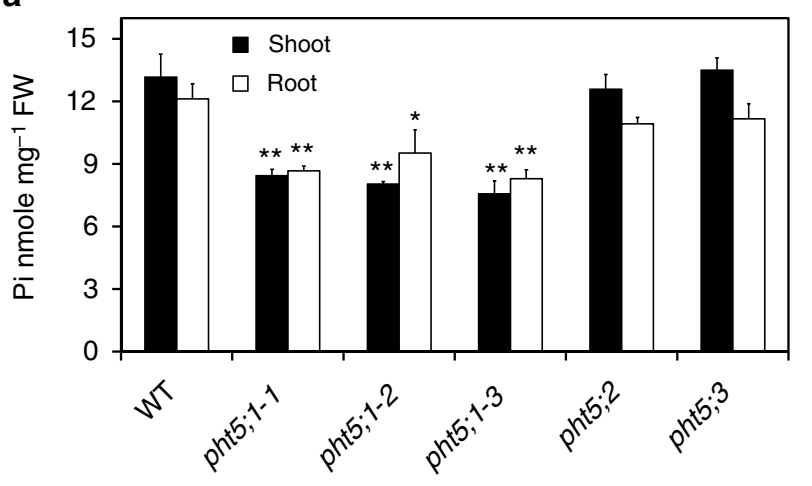

b

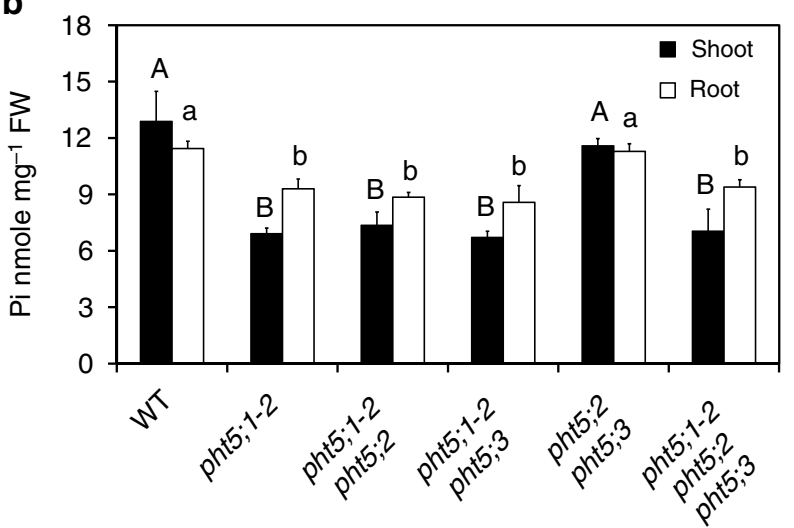

C

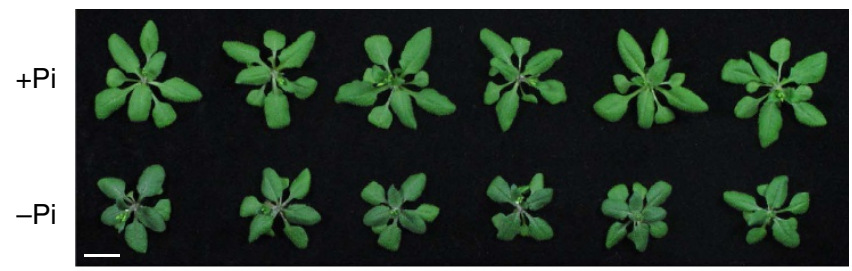

Recovery

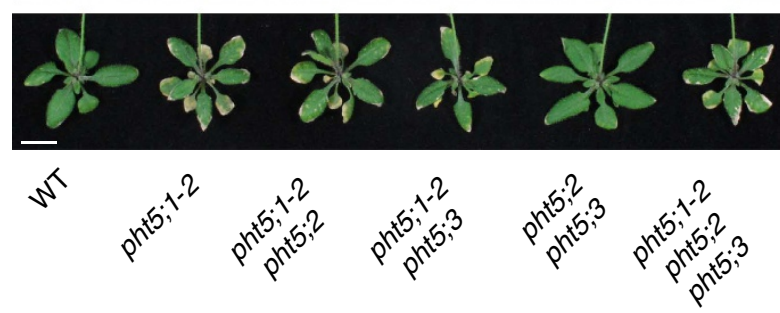

Figure 2 | The capacity of Pi storage and recovery is impaired in the pht5;1 mutants. The Pi level of WT, pht5;1-1, pht5;1-2, pht5;1-3, pht5;2 and pht5;3 plants (a, asterisks indicate a significant difference compared with $\mathrm{WT},{ }^{\star} P<0.05,{ }^{\star}{ }^{\star} P<0.01$, Student's $t$-test) and their respective double and triple mutants ( $\mathbf{b}$, different upper and lower case letters represent a significant difference among genotypes in the shoot and root samples, respectively, analysis of variance $P<0.05$ ) grown under Pi-sufficient conditions. The phenotype of 21-day WT, pht5;1-2, pht5;7-2 pht5;2, pht5;7-2 pht5;3, pht5;2 pht5;3 and pht5;1-2 pht5;2 pht5;3 plants grown under Pi-sufficient $(+\mathrm{P})$ and Pi-deficient ( $-\mathrm{Pi}$ ) conditions, and after 3 days of Pi replenishment following 3 days of Pi starvation (Recovery) (c). Error bar, s.d. $(n=3)$. Scale bars, $10 \mathrm{~mm}$. Results were reproducible in at least three independent experiments.

cytoplasm for each mutant and overexpression line (Fig. 4b). Both the pht5;1-2 and the triple mutants had a reduced vac-Pi level, but an increased cyt-Pi level was only observed for the triple mutant. By contrast, the PHT5-overexpressing lines displayed an increased vac-Pi level, but the PHT5;2-HA-overexpressing lines exhibited a decreased cyt-Pi level. Although the pho 2 mutant also exhibited a higher ratio of vac/cyt Pi $(8.8 \pm 3.3, n=13$, Fig. $4 \mathrm{~b})$, the cyt-Pi level of the pho2 mutant was not significantly different from that of the WT plants (Fig. 4b), suggesting that pho2 is able to maintain $\mathrm{Pi}$ homeostasis at the cellular level by increasing the capacity of vacuolar transport of $\mathrm{Pi}$ at this growth stage. Taken together, these results reinforce the notion that PHT5 proteins mediate the vacuolar storage of $\mathrm{Pi}$ and play a crucial role in the maintenance of cyt-Pi concentrations.

To demonstrate the ability of PHT5 proteins to transport $\mathrm{Pi}$, we expressed AtPHT5;1 in the yeast (Saccharomyces cerevisiae) $v t c 4$ mutant defective in the synthesis and the vacuolar transport of polyphosphate (polyP) ${ }^{35,36}$. We did not detect differences in the Pi transport activity of the isolated vacuoles between the cells expressing AtPHT5;1 and the empty vector controls. This was probably due to the mislocalization or the rapid degradation of the AtPHT5;1-ECFP fusion proteins, as the fluorescence signal was mostly detected inside the vacuole. By contrast, when the rice PHT5;1 homologue (OsSPX-MFS1, Os04g48390) was expressed, the localization of OsSPX-MFS1-ECFP was clearly confined to the tonoplast of yeast cells (Fig. 5a), indicating a proper vacuolar targeting of OsSPX-MFS1. We next isolated vacuoles from the $v t c 4$ mutant expressing OsSPX-MFS1 for Pi uptake assays. After incubation in the ${ }^{33} \mathrm{P}$-labelled $\mathrm{Pi}$ medium, the vacuoles isolated from OsSPX-MFS1-expressing cells exhibited an increased uptake activity of $\mathrm{Pi}$ compared with the empty vector controls, regardless of the presence or the absence of ATP (Fig. 5b). Moreover, the Pi uptake activities were not abolished by the proton ionophore, carbonyl cyanide m-chlorophenyl hydrazone (Fig. 5c). These results indicate that OsSPX-MFS1 facilitates the Pi import to the yeast vacuole independent of ATP and $\mathrm{H}^{+}$gradient.

Similar to the AtPHT5 proteins, GFP-OsSPX-MFS1 also showed a tonoplast localization in Arabidopsis and it restored the low Pi level to the WT level when expressed in pht5;1-2 or pht5;1-3 mutants (Supplementary Fig. 4a,b), suggesting that the Arabidopsis PHT5 proteins and the rice SPX-MFS1 have a similar function in facilitating Pi transport into the plant vacuole.

Altered PHT5 expression disturbs the expression of PSR genes. To evaluate the impact of altered expression of PHT5 family members on $\mathrm{Pi}$ starvation responses, we checked the expression level of a number of known Pi starvation-induced (PSi) genes in the pht5;1-2, the triple mutants, and two 
$\mathbf{a}$

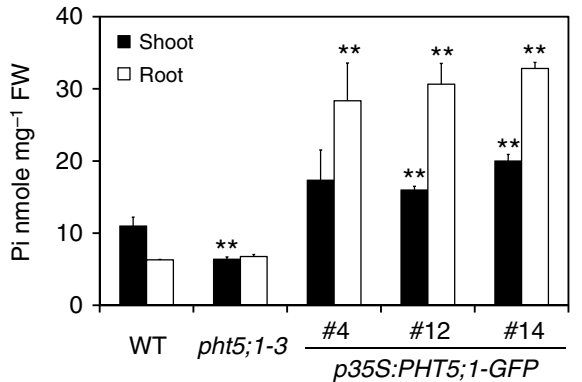

b

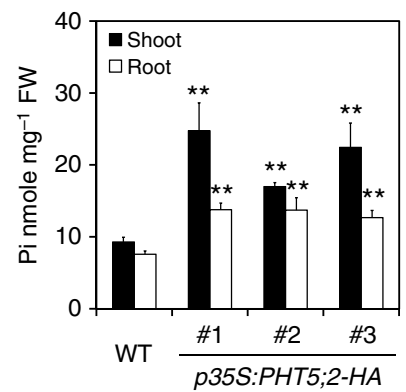

C

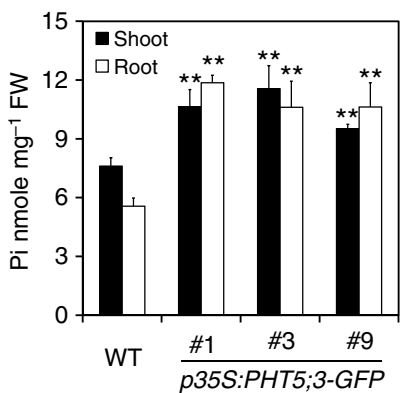

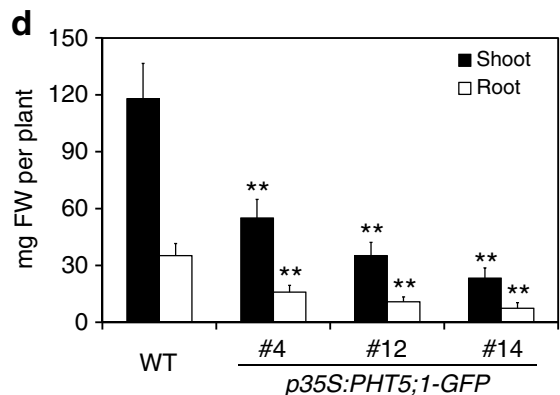

e

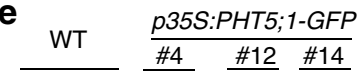

Figure 3 | Overexpression of PHT5 leads to Pi overaccumulation and retarded growth. The Pi level of the shoots and roots of $P H T 5$-overexpressing lines grown under Pi-sufficient conditions. p35S:PHT5;1-GFP in a, p35S:PHT5;2-HA in $\mathbf{b}$ and p35S:PHT5;3-GFP in c. The fresh weight of 21-day-old seedlings (d) and the growth phenotypes of 11-day-old seedlings (e) of PHT5;1-GFP-overexpressing lines. Scale bar, $1 \mathrm{~cm}$. Three independent transgenic lines are shown for


reproducible in at least three independent experiments.

independent PHT5;1-overexpressors by qRT-PCR (Supplementary Fig. 5). Nearly all of the PSi genes examined were upregulated in the PHT5; 1 overexpressors grown under Pi-sufficient conditions. By contrast, these PSi genes were downregulated in the triple mutants, but to a lesser extent in comparison with the PHT5; 1 overexpressors. We therefore focused on the PHT5;1 overexpressors and analysed the transcriptome by RNA-seq analyses (Supplementary Data 1). Two independent PHT5; 1-GFP-overexpressing lines 4 and 12 were examined.

We defined PSR genes as differentially expressed genes, either upregulated or downregulated when setting the $P$-value $<0.05$ based on the analysis of edge $\mathrm{R}^{37}$ and the change of RPKM (reads per kilobase of exon model per million mapped reads) $\geq 2$-fold in the shoot or $\geq 1.5$-fold in the root of WT plants subjected to Pi starvation for 1 or 3 days. Under Pi-sufficient conditions, the gene expression patterns of two PHT5;1-GFP-overexpressing lines showed a positive correlation (Pearson's coefficient of correlation $r=0.73$ ) (Supplementary Fig. 6a). Compared with the WT, the differentially expressed genes in these two lines largely overlapped (Supplementary Fig. 6b). Taking the stronger PHT5;1-GFPoverexpressor 12 for further analyses, we found that 65.1 and $17 \%$ of the WT PSi genes (the PSR genes induced by $\mathrm{Pi}$ starvation in WT) were upregulated, respectively, in the shoot and root of the PHT5;1-GFP overexpressor under Pi-sufficient conditions (Venn diagrams in Fig. 6a,c). Up to 52.3 and $69.1 \%$ of the genes upregulated in the overexpresser relative to the WT, in the shoot and root respectively, were also WT PSi genes (Venn diagrams in Fig. 6a,c). Moreover, 50.4 and $34.0 \%$ of genes downregulated in the overexpresser in the shoot and root, respectively were WT PSr (Pi starvation repressed) genes.

To discern the overall changes of WT PSR genes in the PHT5;1-GFP overexpressor, we compared the gene expression between the overexpressor and WT plants by scatter plots (Fig. 6a-d). Notably, there is a tendency that the PHT5;1-GFP overexpressor had a higher level of expression of PSi genes but a lower level of expression of $\mathrm{PSr}$ genes under $\mathrm{Pi}$-sufficient conditions (Fig. 6a,c). Although the magnitude of the altered gene expression was more evident in the shoot than in the root, they showed a similar trend. Collectively, these results suggest that the overexpression of PHT5;1 triggers Pi starvation-activated transcriptional reprogramming.

Interestingly, under Pi-deficient conditions, we found that the expression level of several of the PSi genes was suppressed in the PHT5;1-GFP overexpressor 12, whereas the expression of several of the PSr genes was upregulated (Fig. 6b,d). Similar results were also observed for the PHT5;1-GFP overexpressor 4 (Supplementary Fig. 6c,d). This may be due to an increased capacity of vac-Pi to buffer the low cyt-Pi in the overexpressors. In addition, a subset of the PSi genes that were downregulated in the root of the PHT5;1-GFP overexpressors under Pi-sufficient conditions were significantly enriched in the gene ontology $(\mathrm{GO})$ categories of the root development (for example, root morphogenesis, root epidermal cell differentiation and trichoblast differentiation) (Fig. 6e). However, these genes were upregulated to an extent comparable to the WT after 3 days of Pi starvation, implying that the internal low cyt-Pi level of the PHT5;1-GFP overexpressors alone was not sufficient to activate these genes. In support of this, the changes of root morphology under low $\mathrm{Pi}$ are mainly associated with the external Pi concentrations ${ }^{38,39}$. Nonetheless, the primary cause for the downregulation of these genes in the PHT5;1-GFP overexpressors under Pi-sufficient conditions is currently not clear.

\section{Discussion}

Similar to other nutrients, Pi has different fates after uptake into the root: (i) it can enter the cytoplasmic pool for the biosynthesis of $\mathrm{P}$-containing compounds or the regulation of protein function 

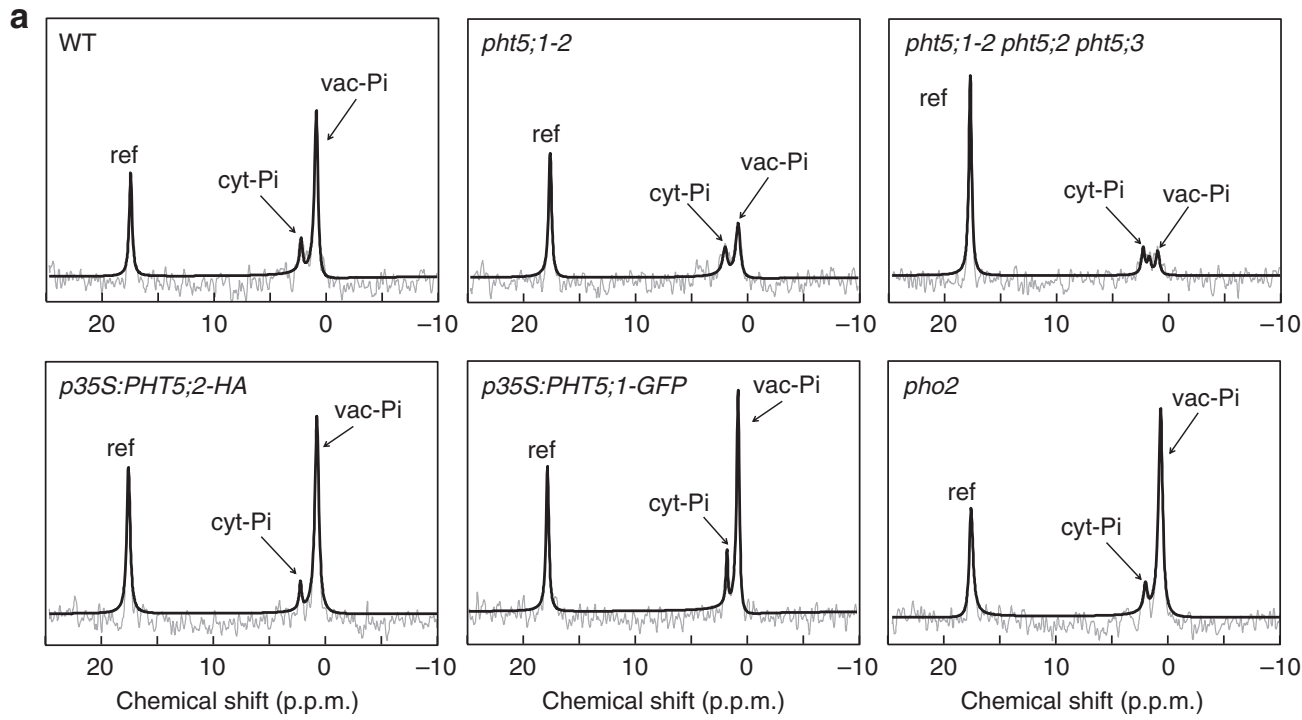

b

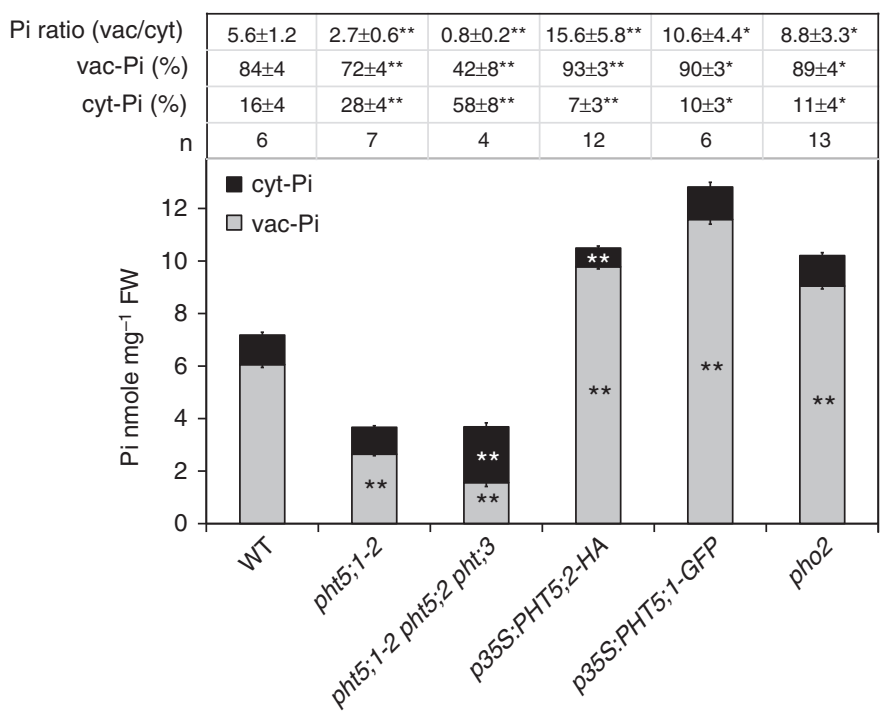

Figure 4 | PHT5 proteins regulate the storage of vac-Pi and cyt-Pi homeostasis. ${ }^{31} \mathrm{P}-\mathrm{MRS}$ analysis of vac-Pi and cyt-Pi (a), $\mathrm{Pi}$ distribution between the vacuole and the cytoplasm, and the calculated vac-Pi and cyt-Pi levels (b) of WT, pht5;1-2, pht5;1-2 pht5;2 pht5;3, p35S:PHT5;2-HA, p35S:PHT5;1-GFP and pho2 seedlings grown under Pi-sufficient conditions. ref, methylene diphosphonate (MDP). Asterisks indicate a significant difference compared with WT, ${ }^{\star} P<0.05$ and ${ }^{\star \star} P<0.01$, Student's $t$-test, \pm s.d.; error bar, s.e. The numbers of biological replicates $(n)$ are indicated in $\mathbf{b}$. Results were combined from three to six independent experiments.
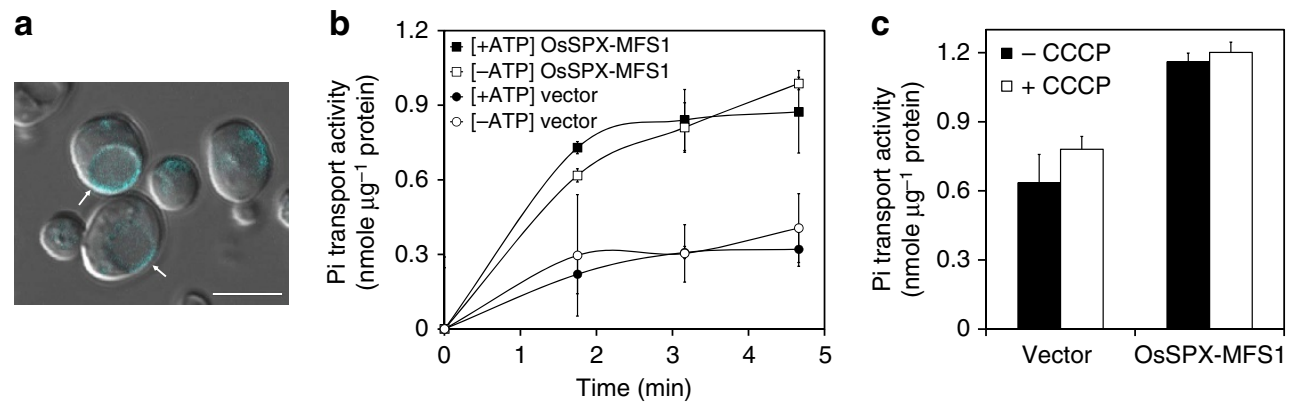

Figure 5 | Phosphate transport activity of rice OsSPX-MFS1 in isolated yeast vacuoles. (a) Fluorescence signal of yeast vtc4 mutant transformed with OsSPX-MFS1-ECFP. Arrows indicate the appearance of fluorescence signals in the tonoplast of yeast. Scale bar, $5 \mu \mathrm{m}$. ${ }^{33} \mathrm{Pi}$ uptake activity of vacuoles isolated from yeast vtc4 mutants transformed with empty vector (pAG415GPD) or expressing OsSPX-MFS1 in the presence of 4 mM ATP [ + ATP] or the absence of ATP [-ATP] at different time points (b) or in the presence of $10 \mu \mathrm{M}$ carbonyl cyanide m-chlorophenyl hydrazone (CCCP) [ + CCCP] or the absence of CCCP $[-\mathrm{CCCP}]$ at $285 \mathrm{~s}$ post incubation $(\mathbf{c})$. Error bar, s.e. $(n=3)$. Results were reproducible in two to four independent experiments. 
and signalling transduction; (ii) it can also be transported radially, passing through several cell layers of the root, towards the vascular bundle for the long-distance translocation to the above-
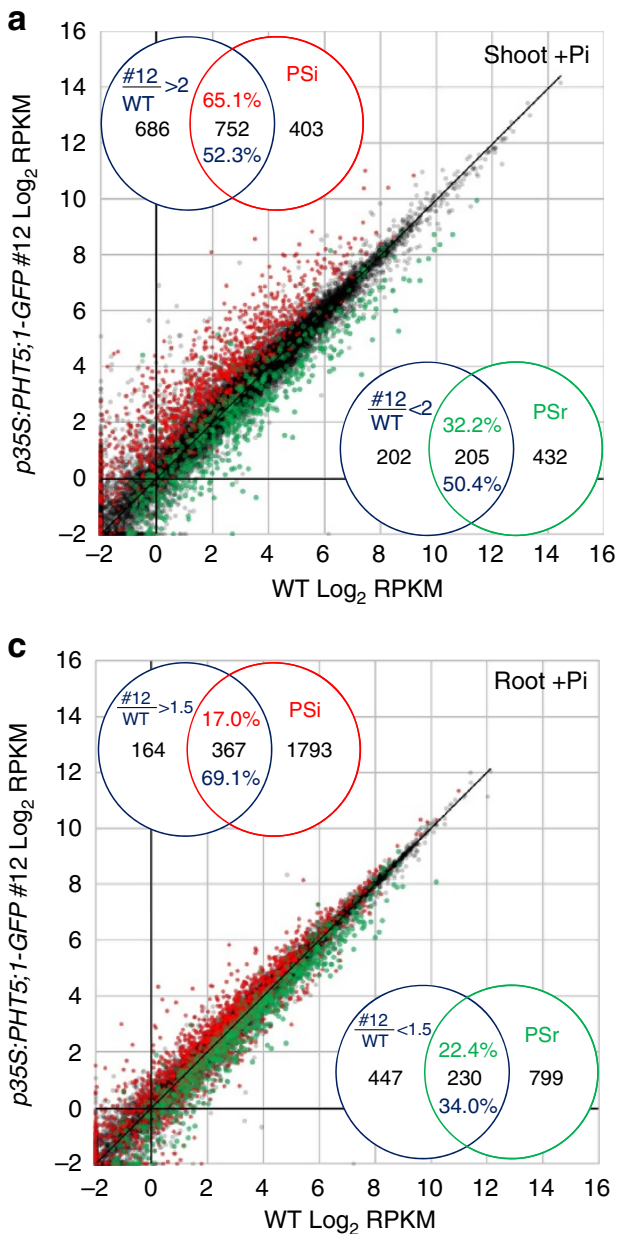

ground tissues; (iii) alternatively, it can enter the vacuole for storage ${ }^{40}$. For decades, ${ }^{31} \mathrm{P}$-NMR spectroscopic monitoring of the exchange of Pi between the cytoplasm and the vacuole suggested
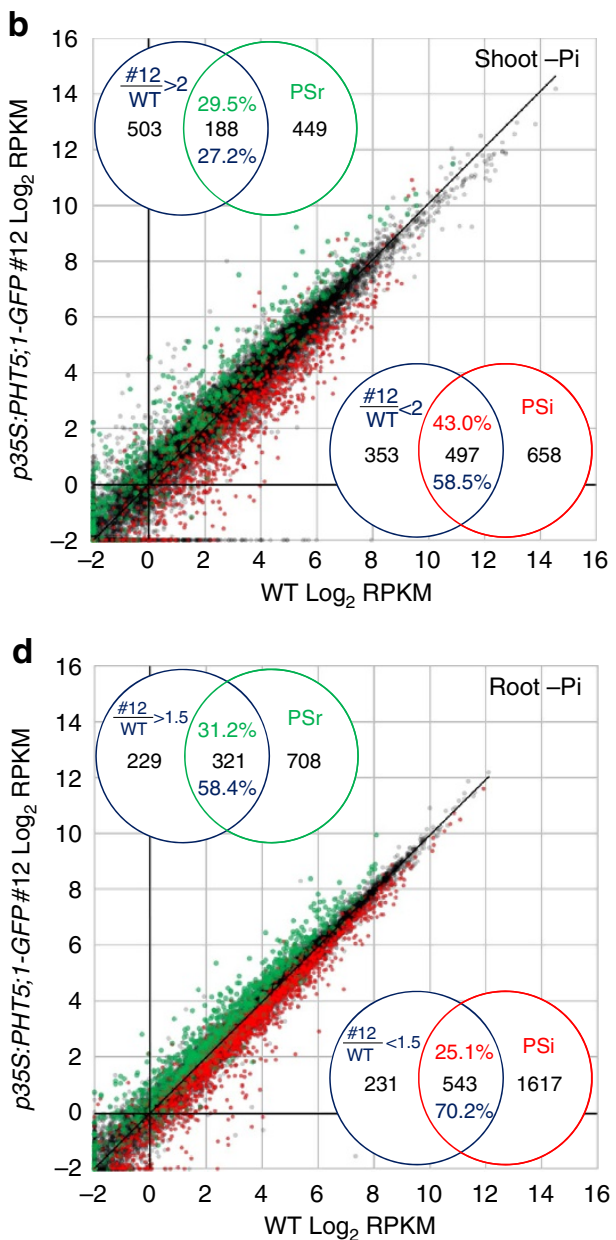

e

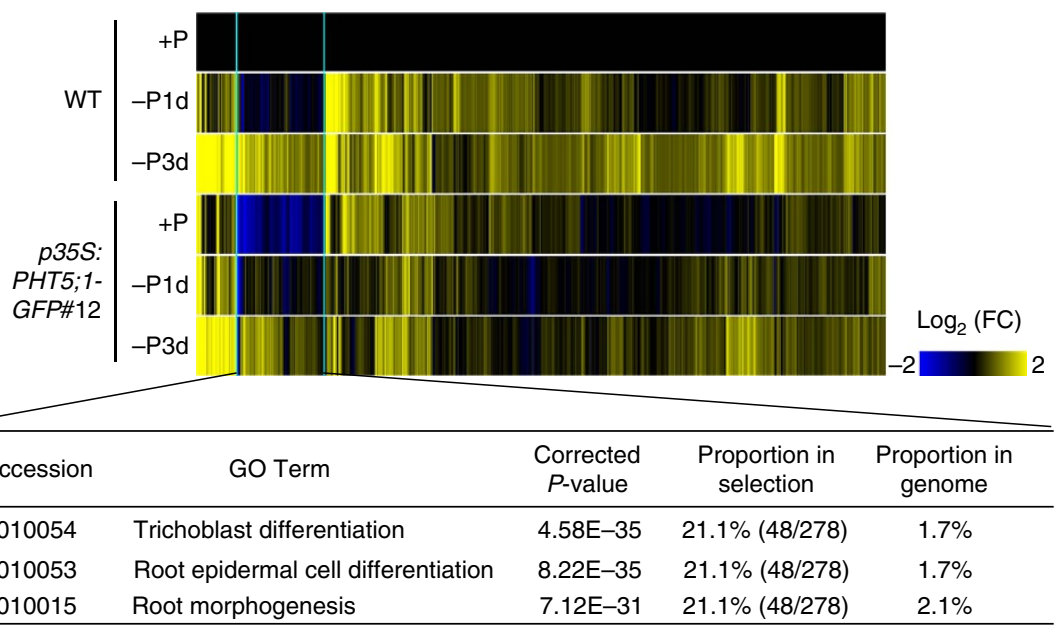

Figure 6 | Overexpression of PHT5; l leads to misregulation of PSR genes. Scatter plots show the comparison of the gene expression (RPKM) in the shoot $(\mathbf{a}, \mathbf{b})$ and root $(\mathbf{c}, \mathbf{d})$ between the PHT5;1-GFP-overexpressing line \#12 and WT plants under Pi-sufficient ( + Pi) (a,c) and 3-day Pi-deficient (-Pi) (b,d) conditions. Each dot represents an individual gene entity. Red and green dots represent, respectively, the PSi and PSr genes with differential regulation during Pi starvation in the WT ( $\geq 2$-fold change in the shoot and $\geq 1.5$-fold change in the root). Venn diagrams in each panel indicate the overlaps of the genes differentially expressed in the PHT5;1-GFP-overexpressing line compared with WT and PSR genes of WT. It is noteworthy that the WT PSi genes were upregulated in the PHT5;1-GFP-overexpressing line under + Pi conditions but downregulated under - Pi conditions. In a similar manner, the WT PSr genes were downregulated in PHT5;1-GFP-overexpressing line under + Pi conditions but upregulated under - Pi conditions. (e) Heat map analyses show the expression of a subset of Pi-starvation-upregulated genes with significant gene ontology (GO) enrichment in the category of root development, which were downregulated in the roots of the PHT5;1-GFP-overexpressing line. P-value was adjusted by Bonferroni correction. 
the existence of vacuolar transport systems ${ }^{41-45}$. However, the mechanism underlying the passage of $\mathrm{Pi}$ across the tonoplast remained obscure. Herein we identified the Arabidopsis SPX-MFS family as the long-sought-after vac-Pi importer and designated them as PHT5 Pi transporters, by phenotypic and functional characterization of the respective PHT5 mutants and overexpressing lines.

Using in vivo ${ }^{31} \mathrm{P}-\mathrm{MRS}$ analysis, we determined the subcellular compartmentation of Pi between the cytoplasm and the vacuole of the Arabidopsis seedlings. Under Pi-replete conditions, the total Pi levels of the single pht5;1 and the triple knockouts were reduced by $40 \%$ relative to the WT plants (Fig. 2a,b); yet, only the triple mutant displayed an increased cytPi level (Fig. 4). In line with the role of PHT5 proteins in facilitating $\mathrm{Pi}$ import across the tonoplast, the PHT5-overexpressing lines overaccumulated $\mathrm{Pi}$ in the vacuole, thus leading to a decreased cyt-Pi level (Fig. 4). Except one report ${ }^{43}$, distinguishing the cytosolic $\mathrm{Pi}$ from the organelle $\mathrm{Pi}$ by ${ }^{31}$ P-NMR analysis has been technically difficult because of the low amount of $\mathrm{Pi}$ and the similar $\mathrm{pH}$ values of these compartments. We thus speculated that the cytosolic $\mathrm{Pi}$ of the PHT5;1-overexpressing lines is even lower than the cyt-Pi level we measured.

Despite the partial redundancy of the three Arabidopsis PHT5 proteins, loss of PHT5;1 rather than PHT5;2 or PHT5;3 led to severe leaf necrosis on Pi replenishment following Pi starvation (Fig. 2c), suggesting that PHT5;1 plays a major role in mediating the $\mathrm{Pi}$ import to the vacuole, preventing $\mathrm{Pi}$ accumulation in the cytoplasm accompanied by massive Pi uptake into the cell. Such a protection mechanism of vac-Pi sequestration is critical for plant growth under varying environmental $\mathrm{Pi}$ status and can explain the tolerance of the pho2 mutant or other Pi overaccumulators to high amounts of cellular $\mathrm{Pi}^{33,34,46,47}$. By contrast, the PHT5overexpressing lines displayed a higher vac/cyt ratio of $\mathrm{Pi}$ compartmentation, a decreased cyt-Pi level and a disturbance of cytoplasmic homeostasis that impaired the plant growth (Figs 3 and 4). Furthermore, given that the triple mutants are viable, we cannot exclude the possibility that plants can exploit other strategies bypassing the PHT5-mediated passage of Pi for their survival.

Although we anticipated to find an altered expression of PHT5 genes in response to varying $\mathrm{Pi}$ supply, in fact the steady-state level of PHT5;1 and PHT5;3 transcripts in the shoot was independent of the Pi status (Fig. 1m,n). Regulation of PHT5 genes at the translational or posttranslational level are likely to be involved. In the Pi-starved root, the reduced expression of PHT5;3 (Fig. 1n) would prevent Pi import into the vacuoles; however, the upregulation of $P H T 5 ; 1$ disagreed with this view (Fig. 1m). In rice, OsSPX-MFS1 and OsSPX-MFS2 (Os02g45520) also showed distinct responses to Pi starvation ${ }^{23,26}$. On transfer to low-Pi medium, the Vtc genes were upregulated and the protein complex was accumulated in yeast vacuolar membranes, to facilitate polyP synthesis and import to the vacuole ${ }^{35,48}$. This led to a lower cyt-Pi level whose feedback stabilized Pi starvation responses for the growth adaptation ${ }^{48}$. We thus speculate that certain Pi-starved plant cells might increase the vac-Pi transport activity to fine-tune the expression of PSR genes by keeping the cyt-Pi level low. Indeed, the vacuoles isolated from Pi-starved barley mesophyll cells showed higher activity of Pi transport than those from Pi-replete cells ${ }^{17}$.

In rice, OsSPX-MFS1 and OsSPX-MFS2 are the targets of a PSi microRNA, OsmiR827 (refs 23,26). AtmiR827 is also induced by Pi starvation in Arabidopsis ${ }^{49}$ and potentially able to cleave the transcript of AtPHT5; 1, although it was not experimentally validated. Instead, AtmiR827 targets another SPX domain-containing protein, NITROGEN LIMITATION ADAPTATION, which regulates the degradation of PHT1 proteins by ubiquitination-mediated endocytosis $^{50}$. Interestingly, AtPHT5; 1 and OsSPX-MFS2 have several transcript variants with different $5^{\prime}$-untranslated region lengths, resulting in differential targeting of miR827.

Our initial attempt to express the Arabidopsis PHT5;1 in yeast cells was unsuccessful, owing to mistargeting or instability of the protein. Instead, we confirmed the capability of the rice OsSPXMFS1 homologue to transport Pi into yeast vacuoles (Fig. 5). We observed that the Pi uptake activities of the OsSPX-MFS1expressing yeast vacuoles was uncoupled from $\mathrm{H}^{+}$and not affected by the presence of ATP (Fig. 5b,c), suggesting that the vac-Pi import is mediated by facilitated diffusion along the electrochemical gradient. The Pi transport into vacuoles isolated from Pi-replete barley leaves was reported to be independent of $\mathrm{ATP}^{17}$. However, ATP stimulated the Pi uptake into the vacuoles isolated from Pi-starved barley leaves and Catharanthus cell culture grown under Pi-sufficient medium, hinting at the requirement of an electrochemical gradient generated by the $\mathrm{H}^{+}$pump ${ }^{17,51}$. It is worth mentioning that we failed to detect a difference in the Pi transport activity of the Arabidopsis intact vacuoles between the WT and the pht5;1 knockout plants. We speculated that unknown cytosolic components required to activate the vac- $\mathrm{Pi}$ transport are missing in this experimental system, or that the Pi influx of the isolated Arabidopsis vacuoles is very low and thus limiting a detailed study ${ }^{51}$.

While this study was under revision, an independent study also reported the role of PHT5;1, which they named VPT1, in mediating vac-Pi storage ${ }^{30}$. Complementary to our observation, the electrophysiological analysis of VPT1-GFP-expressing vacuoles suggested that VPT1 mediates the cytosolic $\mathrm{Pi}$ concentration-dependent $\mathrm{Pi}$ inward current and possesses the single channel-like characteristics ${ }^{30}$. This channel-like property may explain the ATP and $\mathrm{H}^{+}$-independent Pi influx of yeast vacuoles we observed. Liu et al. ${ }^{30}$ also reported that the vpt1 mutant (pht5;1-3 in this study) is more sensitive to high Pi stress when grown in hydroponic medium containing $6.5 \mathrm{mM} \mathrm{Pi}$. However, we did not observe this phenotype, possibly because the concentration is much higher than the Pi-replete growth conditions we used $(250 \mu \mathrm{M} \mathrm{Pi})$. Under our growth conditions, pht5;1-3 mutants showed a reduction of fresh weight by $\sim 20 \%$ compared with WT plants; however, this phenotype was independent of Pi concentrations and was not observed in the other two allelic mutants, pht5;1-1 and pht5;1-2, and even in the pht5;1-2 pht5;2 pht5;3 triple mutant. Nevertheless, all the loss-offunction mutants of PHT5;1, pht5;1-1, pht5;1-2 and pht5;1-3 displayed consistent phenotypes on Pi replenishment.

As the expression of the rice OsSPX-MFS1 complemented the phenotype of Arabidopsis pht5;1 mutants (Supplementary Fig. 4), the Arabidopsis PHT5 proteins and the rice homologue SPX-MFS1 appear to play a similar role in transporting Pi across the tonoplast. However, OsSPX-MFS3 (Os06g03860) was concluded to be a vac-Pi efflux transporter in a recent report based on the influx of $\mathrm{Pi}$ into the Xenopus oocytes in which OsSPX-MFS3 was mislocalized at the plasma membrane ${ }^{27}$. The overexpression of OsSPX-MFS3 led to a decreased Pi level in the rice vacuoles ${ }^{27}$. This seems contradictory to our current findings (and those of Liu et $a l^{30}$ ) of the role of the Arabidopsis PHT5 proteins in vac-Pi storage and sequestration. We then analysed the phylogenetic relationship between 47 SPX-MFS homologues in 13 different species and found that the eudicot and monocot SPX-MFS homologues fall into two different clades (Supplementary Fig. 7). Although the Arabidopsis and rice SPX-MFS homologues share $68-72 \%$ amino acid identity, the result suggests that the monocot and eudicot SPX-MFS genes may diverge before the speciation of monocot and eudicot plants, and 
evolve independently. However, whether the divergence is associated with functional variation ( $\mathrm{Pi}$ export versus $\mathrm{Pi}$ import) requires further validation. Intriguingly, a substantial activity of Pi efflux out of the OsSPX-MFS3-expressing Xenopus oocytes was also detected ${ }^{27}$, hinting the potential capability of OsSPX-MFS3 to transport Pi bidirectionally. How the biophysical properties of PHT5 proteins in these different types of cells contribute to the underlying transport mechanism requires further investigation.

In budding yeast $S$. cerevisiae, the activation of the Pho pathway via intracellular $\mathrm{Pi}$ sensing is at the core of $\mathrm{Pi}$ starvation programme ${ }^{52}$. Despite the unique mechanism of polyP synthesis and storage in the yeast vacuole ${ }^{35,36}$, both the $\mathrm{Pi}$ fluxes at the plasma membrane and at the tonoplast control the amount of cyt- $\mathrm{Pi}^{52,53}$. Molecular mechanisms underlying $\mathrm{Pi}$ sensing, signalling and adaptation in multicellular organisms such as plants are even more complex ${ }^{5}$. Identification of the plant vac-Pi importers allowed us for the first time to address the effect of the internal Pi level on the global transcript expression. From the results of RNA-seq analyses, we propose that the low cyt-Pi level of the PHT5;1-overexpressing lines induces an internal signal of Pi starvation, triggering the genome-wide transcriptional reprogramming resembling the $\mathrm{Pi}$ starvation responses occurring in WT plants. These data suggest that the cyt-Pi level regulates the majority of PSR genes. Although most of the PSR genes were activated in the PHT5;1-overexpressing lines, the expression of a subset of PSR genes associated with trichoblast differentiation was not upregulated but even suppressed, raising the possibility that when the external $\mathrm{Pi}$ is replete, PHT5 proteins play a role in repressing the development of root-hair cells besides their involvement in the maintenance of cyt-Pi homeostasis.

Besides PHT5 proteins, the other eukaryotic SPX domaincontaining Pi transporters such as the yeast Pho87, Pho90, Pho91 and the plant $\mathrm{Pi}$ exporter $\mathrm{PHO} 1$ are also key regulators of $\mathrm{Pi}$ homeostasis ${ }^{19,21,22}$. In yeast, the SPX domain of Pi transporters plays an inhibitory role in regulating the $\mathrm{Pi}$ transport activities through the interaction with the regulatory protein Spl2 (ref. 21). Although many SPX domain-containing proteins were shown to exhibit intrinsic properties required to interact with other proteins $^{21,50,54-57}$, the elucidation of their structural-functional relationship and the identification of novel SPX domaininteracting proteins would be necessary to reveal how the additional cytoplasmic SPX domain modulates the transport activity of PHT5 proteins. Functional studies of different truncated and point mutation variants of PHT5 proteins may reveal more relevant information about their mode of regulation on a molecular basis.

In summary, identification of the Arabidopsis PHT5 family as the vac-Pi importers opens up a new area of investigation into how plants coordinate the vac-Pi transport activity with the cellular metabolism and environmental fluctuations. Our findings also invoke interesting questions regarding how $\mathrm{Pi}$ is translocated out of the vacuole for metabolic needs on Pi deficiency and during $\mathrm{Pi}$ remobilization. The rice OsSPX-MFS3 was recently shown to be responsible for the vac-Pi export ${ }^{27}$; however, our results here support that its paralogue (OsSPX-MFS1) and orthologues (Arabidopsis PHT5 proteins) contribute to the Pi import into vacuoles. In the future, identification of both $\mathrm{Pi}$ influx and efflux systems in the same plant species can offer clear and insightful information.

\section{Methods}

Growth conditions and plant material. Arabidopsis thaliana ecotype Columbia (Col-0) was used in this study. The pht5;1-1 (SAIL_789_E03), pht5;1-2 (SAIL_96_H01), pht5;1-3 (SALK_006647), pht5;2 (SALK_009309) and pht5;3 (SAIL_422_D07) T-DNA insertion mutants were obtained from the Arabidopsis
Stock Center. Seeds were surface sterilized and germinated on agar plates with one-half modified Hoagland nutrient solution containing $250 \mu \mathrm{M} \mathrm{K \textrm {H } _ { 2 }} \mathrm{PO}_{4}, 1 \%$ sucrose and $1 \%$ bactoagar. The Pi-sufficient $(+\mathrm{Pi})$ and Pi-deficient $(-\mathrm{Pi})$ media were supplemented with 250 and $10 \mu \mathrm{M} \mathrm{KH}_{2} \mathrm{PO}_{4}$, respectively, unless specified otherwise. For hydroponic growth, the medium was prepared without supplement of sucrose. For the plant samples used for ${ }^{31} \mathrm{P}-\mathrm{MRS}$ analysis, $20 \mathrm{mg}$ of seeds were surface sterilized and evenly distributed on the surface of the $100-\mu \mathrm{m}$ nylon mesh cell drainer (BD 352360) by mixing with sterilized 1\% agarose. The seeds were germinated in the sterilized six-well culture plates, each containing $5 \mathrm{ml}$ of one-half modified Hoagland nutrient solution with $1 \mathrm{mM} \mathrm{KH}_{2} \mathrm{PO}_{4}$ and $1 \%$ sucrose. Three days after germination, the growth medium was exchanged at $12-24 \mathrm{~h}$ intervals. For RNA-seq, sample preparations are described as follows: 10-day-old seedlings were germinated and grown under $+\mathrm{Pi}$ conditions on the agar plates and then transferred to the $-\mathrm{Pi}$ media for an additional 1-3 days.

Gene constructions and plant transformation. All the insert fragments of interest were amplified and cloned into pCR8/GW/TOPO (Invitrogen) for sequencing and then recombined into the desired Gateway destination vectors via LR Clonase enzyme mix (Invitrogen). For Arabidopsis overexpression experiments, the open reading frame (ORF) of PHT5;1, PHT5;3 and OsSPX-MFS1 (Os04g48390) complementary DNA and PHT5;2 from genomic DNA were cloned and recombined into the Gateway destination vector pK7FWG2.0 or pMDC32, designated as p35S:PHT5;1-GFP, p35S:PHT5;3-GFP, p35S:GFP-OsSPX-MFS1 and p35S:PHT5;2$H A$. For the promoter assay, $p P H T 5$ :GUS constructs were generated using pMDC162 as the Gateway destination vector. For the complementation of pht5;1-3 mutants, the PHT5;1 genomic sequence was cloned and recombined into the Gateway destination vector pGWB504. Transgenic plants were generated using an Agrobacterium tumefaciens dipping procedure ${ }^{58}$ via strain GV3101.

Transient expression. Transformation of Arabidopsis mesophyll protoplasts for transient expression of fluorescence fusion proteins was performed with tape-Arabidopsis sandwich method ${ }^{59}$. The Agrobacterium-mediated transient expression in tobacco leaves was conducted ${ }^{55}$. Agrobacterium EHA105 strain harbouring the constructs of genes of interest was grown in Luria-Bertani medium, collected and resuspended in the infiltration medium $(10 \mathrm{mM} \mathrm{MgCl}, 10 \mathrm{mM} \mathrm{MES}$ and $100 \mu \mathrm{M}$ acetosyringone) to an $\mathrm{OD}_{600}$ of 1.0. The second or third true leaves of 3-week-old tobacco plants was infiltrated with Agrobacterium and analysed at 3 days post infiltration. Confocal microscopy images were taken using a Zeiss LSM 510 META NLO DuoScan with objectives LCI Plan-Neofluar $\times 63 / 1.3 \mathrm{Imm}$ and Plan-Apochromat $\times 100 / 1.4$ oil. Excitation/emission wavelengths were $488 \mathrm{~nm} / 495-510 \mathrm{~nm}$ for GFP.

GUS staining. GUS activity was detected by a modified method ${ }^{60}$. Briefly, transgenic plants were vacuum infiltrated with solution containing $1.9 \mathrm{mM} \mathrm{X}$-Gluc (5-bromo-4-chloro-3-indoyl- $\beta$-D-glucuronide) for 4 to $6 \mathrm{~h}$ and chlorophyll was then cleared from the sample by $75 \%$ ethanol after staining. At least ten independent transgenic lines for each construct were examined. Representative results were shown.

Measurement of phosphate contents. Tissues were homogenized with $1 \%$ glacial acetic acid. After centrifugation, the supernatant was collected and mixed with assay solution containing $0.35 \% \mathrm{NH}_{4} \mathrm{MoO}_{4}, 0.86 \mathrm{~N} \mathrm{H}_{2} \mathrm{SO}_{4}$ and $1.4 \%$ ascorbic acid. $\mathrm{Pi}$ contents were determined by colorimetric assay based on the formation of phosphomolybdate followed by its reduction with ascorbic acid ${ }^{31}$.

RNA isolation and qRT-PCR. Total RNA was isolated using RNAzol (Molecular Research Center) followed by treatment with DNase I (Ambion), to eliminate genomic DNA contamination. cDNA was synthesized from 0.5 to $1 \mu \mathrm{g}$ total RNA using Moloney murine leukemia virus reverse transcriptase (Invitrogen) with oligo(dT) primer. qRT-PCR was performed using the Power SYBR Green PCR Master Mix kit (Applied Biosystems) on a 7300 Real-Time PCR system (Applied Biosystems) according to the manufacturer's instructions. Relative expression levels were normalized to UBQ10 as an internal control. For RNA-seq, RNA quality was verified by Agilent Bioanalyzer 2100 and only samples with RNA integrity numbers above 9.0 were used. Oligonucleotide sequences of the primer pairs used for PCR and cloning are shown in Supplementary Table 1.

High-throughput RNA-seq analysis. Two biological replicates per condition and about 20 seedlings per replicates were used for library synthesis. Twelve libraries were multiplexed and loaded on each lane of the Illumina Hiseq flow cell v3. Sequencing was then performed on a Hiseq 2500, as a $2 \times 100$ paired-end run, according to the manufacturer's protocol (Illumina). The trimmed paired reads from each sample were mapped against the A. thaliana reference (TAIR10) using CLC Genomics Workbench (CLC Bio, http://www.clcbio.com), in which the expression values were reported as RPKM. An average of $96 \%$ of trimmed reads equivalent to 17-33 million reads per library was successfully mapped to the genome. For statistical analysis, the software package edgeR (empirical analysis of 
DGE in R ${ }^{37}$ implanted in CLC Genomics Workbench was employed. The exon mapped reads for individual gene were compared and false discovery rate-corrected $P$-values was calculated. Total count filter cutoff is set at 5 using the Estimate tagwise dispersions. The entities with RPKM value $<0.25$ were assigned to 0.25 for calculation of fold changes. For differential expression comparisons, false discovery rate-corrected $P$-value $<0.05$ coupled with fold changes of RPKM was applied to filter out differentially expressed genes.

${ }^{31}$ P-MRS analysis. ${ }^{31} \mathrm{P}-\mathrm{MRS}$ data were acquired using a 7T MRI system (ClinScan 70/30 USR, Bruker, Ettlingen, Germany) with a dual tuned 1H/31P Radiofrequency coil. Eight-day-old seedlings together with the cell drainer were rinsed with deionized distilled water. Next, the whole sample was immersed under $20 \mathrm{ml}$ perfusion buffer $\left(10 \mathrm{mM}\right.$ MOPS $\mathrm{pH}=7.5,50 \mathrm{mM}$ glucose and $\left.0.1 \mathrm{mM} \mathrm{CaSO}_{4}\right)$ in a glass beaker and then placed above the centre of the coil. One hundred microlitres of $25 \mathrm{mM}$ methylene diphosphonate (MDP) was used as an internal reference of chemical shift $(\delta)$, the value corresponding 17.9 p.p.m. relative to $85 \%$ phosphate solution (0 p.p.m.). The positioning of plant samples, MDP reference and Radiofrequency coil were kept consistent among all the experiments.

Before the acquisition of phosphorus data, magnetic field homogeneity was shimmed over $25 \times 25 \times 20 \mathrm{~mm}^{3}$ volume based on the ${ }^{1} \mathrm{H}$ signal from the sample. To ensure the homogeneity of the magnetic field, the shimming procedure was repeated until the linewidth of the water peak was $<10 \mathrm{~Hz}$. The shimming setting was then used for acquisition of phosphorus spectroscopic data. ${ }^{31} \mathrm{P}$ data were collected using a single hard pulse sequence with free induction decay acquisition with the following parameters: bandwidth $=6,002 \mathrm{~Hz}$, repetition time $(\mathrm{TR})=2,000 \mathrm{~ms}$ and number of averages $=2,048$. The scanning time on the ${ }^{31} \mathrm{P}$ spectroscopy of each sample was $68.3 \mathrm{~min}$. To examine the $\mathrm{pH}$ values of the observed vacuolar and cyt-Pi peaks, a $\mathrm{pH}$ calibration curve was obtained by using a series of $1 \mathrm{M}$ phosphate solutions with various $\mathrm{pH}$ values from 4.8 to 7.4 . All ${ }^{31} \mathrm{P}$ data were quantified using the time-domain fitting software (jMRUI) ${ }^{61}$ with the AMARES algorithm. Spectral process included $35 \mathrm{~Hz}$ Gaussian apodization, automatic zero-order phase adjustment and the first-order phase adjustment with range fixed within -1 and 1 . The resonances corresponding to the peak of vac- $\mathrm{Pi}$ $(0.82-1.27$ p.p.m.) and the cyt-Pi (cyt-Pi, 2.08-2.62 p.p.m.) were quantified. The ratios of vac-Pi to cyt-Pi were calculated based on jMRUI quantification results.

OsSPX-MFS1 expression in yeast and vacuole transport assay. The ORF of OsSPX-MFS1 was cloned and recombined into the Gateway destination vector pAG415GPD-ccdB and transformed into $S$. cerevisiae strain $v t c 4$ mutant ${ }^{36} \cdot{ }^{33} \mathrm{P}$ transport assay was performed with isolated yeast vacuoles according to a modified protocol ${ }^{62}$. After incubation with ${ }^{33} \mathrm{Pi}$, vacuoles were floated and purified with silicon il $^{63}$ followed by radioactivity measurement. When applied, a final concentration of $4 \mathrm{mM}$ ATP or $10 \mu \mathrm{M}$ carbonyl cyanide m-chlorophenyl hydrazone (CCCP) was added in the uptake solution. The ${ }^{33} \mathrm{Pi}$ uptake value at the zero-time point was used for the subtraction of background. To analyse the subcellular localization of OsSPX-MFS1, the ORF of OsSPX-MFS1 without a stop codon was cloned into pAG415GPD-ccdB-eCFP, to fuse with enhanced cyan fluorescent protein (ECFP) at the C terminus. The fluorescent signal was observed by confocal microscopy.

\section{References}

1. Kirkby, E. in Marschner's Mineral Nutrition of Higher Plants 3rd Edn (ed. Marschner, P.) 3-5 (Academic Press, 2012).

2. Neumann, G. \& Römheld, V. in Marschner's Mineral Nutrition of Higher Plants 3rd Edn (ed. Marschner, P.) 347-368 (Academic Press, 2012).

3. Manning, D. A. C. Phosphate minerals, environmental pollution and sustainable agriculture. Elements 4, 105-108 (2008).

4. Gilbert, N. Environment: the disappearing nutrient. Nature 461, 716-718 (2009).

5. Chiou, T. J. \& Lin, S. I. Signaling network in sensing phosphate availability in plants. Annu. Rev. Plant Biol. 62, 185-206 (2011).

6. Nussaume, L. et al. Phosphate import in plants: focus on the PHT1 transporters. Front. Plant. Sci. 2, 83 (2011).

7. Zhu, W. et al. The mitochondrial phosphate transporters modulate plant responses to salt stress via affecting ATP and gibberellin metabolism in Arabidopsis thaliana. PLoS ONE 7, e43530 (2012).

8. Hassler, S. et al. Lack of the Golgi phosphate transporter PHT4;6 causes strong developmental defects, constitutively activated disease resistance mechanisms and altered intracellular phosphate compartmentation in Arabidopsis. Plant J. 72, 732-744 (2012)

9. Irigoyen, S., Karlsson, P. M., Kuruvilla, J., Spetea, C. \& Versaw, W. K. The sinkspecific plastidic phosphate transporter PHT4;2 influences starch accumulation and leaf size in Arabidopsis. Plant Physiol. 154, 1765-1777 (2011).

10. Cubero, B. et al. The phosphate transporter PHT4;6 is a determinant of salt tolerance that is localized to the Golgi apparatus of Arabidopsis. Mol. Plant 2, 535-552 (2009).

11. Guo, B., Irigoyen, S., Fowler, T. B. \& Versaw, W. K. Differential expression and phylogenetic analysis suggest specialization of plastid-localized members of the
PHT4 phosphate transporter family for photosynthetic and heterotrophic tissues. Plant Signal. Behav. 3, 784-790 (2008).

12. Versaw, W. K. \& Harrison, M. J. A chloroplast phosphate transporter, PHT2; 1 , influences allocation of phosphate within the plant and phosphate-starvation responses. Plant Cell 14, 1751-1766 (2002).

13. Marty, F. Plant vacuoles. Plant Cell 11, 587-599 (1999)

14. Bieleski, R. L. Phosphate pools, phosphate transport, and phosphate availability. Annu. Rev. Plant Physiol. 24, 225-252 (1973).

15. Mimura, T. Regulation of phosphate transport and homeostasis in plant cells. Int. Rev. Cytol. 191, 149-200 (1999).

16. Bieleski, R. L. \& Ferguson, I. B. in Inorganic Plant Nutrition. Encyclopedia of Plant Physiology Vol. 15, 422-429 (Springer-Verlag, Berlin, 1983).

17. Mimura, T. et al. Phosphate transport across biomembranes and cytosolic phosphate homeostasis in barley leaves. Planta 180, 139-146 (1990).

18. Rao, I. M. \& Terry, N. Leaf phosphate status, photosynthesis, and carbon partitioning in sugar beet (IV. Changes with time following increased supply of phosphate to low-phosphate plants). Plant Physiol. 107, 1313-1321 (1995).

19. Secco, D. et al. The emerging importance of the SPX domain-containing proteins in phosphate homeostasis. New Phytol. 193, 842-851 (2012).

20. Secco, D., Wang, C., Shou, H. \& Whelan, J. Phosphate homeostasis in the yeast Saccharomyces cerevisiae, the key role of the SPX domain-containing proteins. FEBS Lett. 586, 289-298 (2012).

21. Hürlimann, H. C., Pinson, B., Stadler-Waibel, M., Zeeman, S. C. \& Freimoser, F. M. The SPX domain of the yeast low-affinity phosphate transporter Pho 90 regulates transport activity. EMBO Rep. 10, 1003-1008 (2009).

22. Hürlimann, H. C., Stadler-Waibel, M., Werner, T. P. \& Freimoser, F. M. Pho91 is a vacuolar phosphate transporter that regulates phosphate and polyphosphate metabolism in Saccharomyces cerevisiae. Mol. Biol. Cell 18, 4438-4445 (2007).

23. Lin, S.-I. et al. Complex regulation of two target genes encoding SPX-MFS proteins by rice miR827 in response to phosphate starvation. Plant Cell Physiol. 51, 2119-2131 (2010).

24. Duan, K. et al. Characterization of a sub-family of Arabidopsis genes with the SPX domain reveals their diverse functions in plant tolerance to phosphorus starvation. Plant J. 54, 965-975 (2008).

25. Marger, M. D. \& Saier, M. H. A major superfamily of transmembrane facilitators that catalyse uniport, symport and antiport. Trends Biochem. Sci. 18, 13-20 (1993).

26. Wang, C. et al. Functional characterization of the rice SPX-MFS family reveals a key role of OsSPX-MFS1 in controlling phosphate homeostasis in leaves. New Phytol. 196, 139-148 (2012).

27. Wang, C. et al. Rice SPX-Major Facility Superfamily3, a vacuolar phosphate efflux transporter, is involved in maintaining phosphate homeostasis in rice. Plant Physiol. 169, 2822-2831 (2015).

28. Poirier, Y. \& Bucher, M. Phosphate transport and homeostasis in Arabidopsis. Arabidopsis Book 1, e0024 (2002).

29. Rausch, C. \& Bucher, M. Molecular mechanisms of phosphate transport in plants. Planta 216, 23-37 (2002).

30. Liu, J. et al. A vacuolar phosphate transporter essential for phosphate homeostasis in Arabidopsis. Proc. Natl Acad. Sci. USA 112, E6571-E6578 (2015).

31. Chiou, T. J. et al. Regulation of phosphate homeostasis by microRNA in Arabidopsis. Plant Cell 18, 412-421 (2006).

32. Roberts, J. K. M., Ray, P. M., Wade-Jardetzky, N. \& Jardetzky, O. Estimation of cytoplasmic and vacuolar $\mathrm{pH}$ in higher plant cells by ${ }^{31} \mathrm{P}$ NMR. Nature 283, 870-872 (1980).

33. Delhaize, E. \& Randall, P. J. Characterization of a phosphate-accumulator mutant of Arabidopsis thaliana. Plant Physiol. 107, 207-213 (1995).

34. Aung, K. et al. pho2, a phosphate overaccumulator, is caused by a nonsense mutation in a microRNA399 target gene. Plant Physiol. 141, 1000-1011 (2006).

35. Hothorn, M. et al. Catalytic Core of a Membrane-associated eukaryotic polyphosphate polymerase. Science 324, 513-516 (2009).

36. Ogawa, N., DeRisi, J. \& Brown, P. O. New components of a system for phosphate accumulation and polyphosphate metabolism in Saccharomyces cerevisiae revealed by genomic expression analysis. Mol. Biol. Cell 11, 4309-4321 (2000).

37. Robinson, M. D., McCarthy, D. J. \& Smyth, G. K. edgeR: a Bioconductor package for differential expression analysis of digital gene expression data. Bioinformatics 26, 139-140 (2010).

38. Liu, T. Y., Chang, C. Y. \& Chiou, T. J. The long-distance signaling of mineral macronutrients. Curr. Opin. Plant Biol. 12, 312-319 (2009).

39. Thibaud, M. C. et al. Dissection of local and systemic transcriptional responses to phosphate starvation in Arabidopsis. Plant J. 64, 775-789 (2010).

40. Schachtman, D. P., Reid, R. J. \& Ayling, S. M. Phosphorus uptake by plants: from soil to cell. Plant Physiol. 116, 447-453 (1998). 
41. Tu, S. I., Cavanaugh, J. R. \& Boswell, R. T. Phosphate uptake by excised maize root tips studied by in vivo ${ }^{31} \mathrm{P}$ nuclear magnetic resonance spectroscopy. Plant Physiol. 93, 778-784 (1990).

42. Lee, R. B., Ratcliffe, R. G. \& Southon, T. E. ${ }^{31}$ P NMR measurements of the cytoplasmic and vacuolar Pi content of mature maize roots: relationships with phosphorus status and phosphate fluxes. J. Exp. Bot. 41, 1063-1078 (1990).

43. Pratt, J. et al. Phosphate (Pi) starvation effect on the cytosolic Pi concentration and $\mathrm{Pi}$ exchanges across the tonoplast in plant cells: an in vivo ${ }^{31} \mathrm{P}$-nuclear magnetic resonance study using methylphosphonate as a Pi analog. Plant Physiol. 151, 1646-1657 (2009).

44. Rebeille, F., Bligny, R., Martin, J. B. \& Douce, R. Relationship between the cytoplasm and the vacuole phosphate pool in Acer pseudoplatanus cells. Arch. Biochem. Biophys. 225, 143-148 (1983).

45. Brodelius, P. \& Vogel, H. J. A phosphorus-31 nuclear magnetic resonance study of phosphate uptake and storage in cultured Catharanthus roseus and Daucus carota plant cells. J. Biol. Chem. 260, 3556-3560 (1985).

46. Liu, T. Y. et al. Vacuolar $\mathrm{Ca}^{2+} / \mathrm{H}^{+}$transport activity is required for systemic phosphate homeostasis involving shoot-to-root signaling in Arabidopsis. Plant Physiol. 156, 1176-1189 (2011).

47. Kuo, H. F. et al. Arabidopsis inositol pentakisphosphate 2-kinase, AtIPK1, is required for growth and modulates phosphate homeostasis at the transcriptional level. Plant J. 80, 503-515 (2014).

48. Vardi, N. et al. Sequential feedback induction stabilizes the phosphate starvation response in budding yeast. Cell Rep. 6, 1122-1134 (2014).

49. Hsieh, L. C. et al. Uncovering small RNA-mediated responses to phosphate deficiency in Arabidopsis by deep sequencing. Plant Physiol. 151, 2120-2132 (2009).

50. Lin, W. Y., Huang, T. K. \& Chiou, T. J. NITROGEN LIMITATION ADAPTATION, a target of microRNA827, mediates degradation of plasma membrane-localized phosphate transporters to maintain phosphate homeostasis in Arabidopsis. Plant Cell 25, 4061-4074 (2013).

51. Massonneau, A., Martinoia, E., Dietz, K. J. \& Mimura, T. Phosphate uptake across the tonoplast of intact vacuoles isolated from suspension-cultured cells of Catharanthus roseus (L.) G. Don. Planta 211, 390-395 (2000).

52. Auesukaree, C. et al. Intracellular phosphate serves as a signal for the regulation of the PHO pathway in Saccharomyces cerevisiae. J. Biol. Chem. 279, 17289-17294 (2004)

53. Mouillon, J. M. \& Persson, B. L. New aspects on phosphate sensing and signalling in Saccharomyces cerevisiae. FEMS. Yeast Res. 6, 171-176 (2006).

54. Zhou, Y. \& Ni, M. SHORT HYPOCOTYL UNDER BLUE1 truncations and mutations alter its association with a signaling protein complex in Arabidopsis. Plant Cell 22, 703-715 (2010).

55. Liu, T. Y. et al. PHO2-dependent degradation of PHO1 modulates phosphate homeostasis in Arabidopsis. Plant Cell 24, 2168-2183 (2012).

56. Puga, M. I. et al. SPX1 is a phosphate-dependent inhibitor of Phosphate Starvation Response 1 in Arabidopsis. Proc. Natl Acad. Sci. USA 111, 14947-14952 (2014)

57. Wang, Z. et al. Rice SPX1 and SPX2 inhibit phosphate starvation responses through interacting with PHR2 in a phosphate-dependent manner. Proc. Natl Acad. Sci. USA 111, 14953-14958 (2014).

58. Clough, S. J. \& Bent, A. F. Floral dip: a simplified method for Agrobacterium-mediated transformation of Arabidopsis thaliana. Plant J. 16, 735-743 (1998).

59. Wu, F. H. et al. Tape-Arabidopsis Sandwich - a simpler Arabidopsis protoplast isolation method. Plant Methods 5, 16 (2009).

60. Lin, S. I. et al. Differential regulation of FLOWERING LOCUS C expression by vernalization in cabbage and Arabidopsis. Plant Physiol. 137, 1037-1048 (2005).
61. Naressi, A., Couturier, C., Castang, I., de Beer, R. \& Graveron-Demilly, D. Java-based graphical user interface for MRUI, a software package for quantitation of in vivo/medical magnetic resonance spectroscopy signals. Comput. Biol. Med. 31, 269-286 (2001).

62. Russnak, R., Konczal, D. \& McIntire, S. L. A family of yeast proteins mediating bidirectional vacuolar amino acid transport. J Biol Chem. 276, 23849-23857 (2001).

63. Rodrigues, J. et al. Flow cytometry as a novel tool for structural and functional characterization of isolated yeast vacuoles. Microbiology 159, 848-856 (2013).

\section{Acknowledgements}

This work was supported by grants from the Ministry of Science and Technology of the Republic of China (NSC 102-2321-B-001-047, MOST 103-2321-B-001-034 and MOST 104-2321-B-001-013) and Academia Sinica, Taiwan (AS-103-TP-B11 and AS-103-SSA03). S.-Y.Y. is a recipient of a postdoctoral fellowship from Academia Sinica. We thank Chun-Yi Chen and Ya-Ting Chao for assistance with RNA-seq analysis, and Yu-Ying Hsu, Ching-Mei Sun, Jia-Ling Li and Yen-Yu Lin for assistance with Pi transport assay. We thank Doris Rentsch and Marianne Suter Grotemeyer (University of Bern, Switzerland) for assistance with the establishment of the yeast vacuole isolation protocol, and Shu-Chen Shen from the Scientific Instrument Center of Academia Sinica for assistance with the confocal microscopy analysis. We also thank the High Throughput Genomics Core Facility of the Biodiversity Research Center in Academia Sinica, Taiwan, for RNA-seq library construction and next-generation sequencing experiments and the Center for Advanced Molecular Imaging and Translation at Chang Gung Memorial Hospital, Linkou, for providing the instrument for MRS analyses. We gratefully acknowledge Enrico Martinoia (University of Zurich, Switzerland) for helping the Pi uptake assay using Arabidopsis intact vacuoles and constructive discussion about the results.

\section{Author contributions}

T.-J.C. designed the project. T.-Y.L., T.-K.H., S.-Y.Y., Y.-T.H., S.-F.C. and W.-C.L. performed the experiments and analysed the data. S.-M.H., F.-N.W. and S.-Y.T. analysed the data and wrote the manuscript. T.-Y.L., T.-K.H., S.-Y.Y. and T.-J.C. contributed to the study design during the project course and wrote the manuscript.

\section{Additional information}

Accession codes: The RNA-seq data have been deposited in the NCBI Gene Expression Omnibus under accession code GSE74856.

Supplementary Information accompanies this paper at http://www.nature.com/ naturecommunications

Competing financial interests: The authors declare no competing financial interests.

Reprints and permission information is available online at http://npg.nature.com/ reprintsandpermissions/

How to cite this article: Liu, T.-Y. et al. Identification of plant vacuolar transporters mediating phosphate storage. Nat. Commun. 7:11095 doi: 10.1038/ncomms11095 (2016).

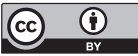

This work is licensed under a Creative Commons Attribution 4.0 International License. The images or other third party material in this article are included in the article's Creative Commons license, unless indicated otherwise in the credit line; if the material is not included under the Creative Commons license, users will need to obtain permission from the license holder to reproduce the material. To view a copy of this license, visit http://creativecommons.org/licenses/by/4.0/ 